\title{
Compressive behaviour of very dense structured granular geo-materials
}

\author{
Mohammad Khoshini ${ }^{1,}{ }^{*}$, Arman Khoshghalb ${ }^{1}$, and Nasser Khalili ${ }^{1}$ \\ ${ }^{1}$ School of Civil and Environmental Engineering, UNSW Sydney, Australia
}

\begin{abstract}
The isotropic compression behaviour of dense structured geo-materials and the associated degradation at failure is addressed in a non-qualitative manner. To this end, the general behaviour of fully de-structured geo-materials, e.g. sands, as an accepted reference is thoroughly investigated. The parameters affecting the behaviour of de-structured materials such as mineralogy, gradation and fines content, and relative density are discussed. The isotropic compression behaviour of a weathered weak sandstone, representative of a structured granular geo-material, is then investigated along the isotropic compression stress path under a range of pressures from nil to $100 \mathrm{MPa}$. Both structured and fully destructured states of the material are tested implementing the proposed quantification method. The effect of structure on the compressibility of the material is found to be tangible. By plotting the specific volume versus natural log of the mean effective stress, the onset of structure collapse and the successive degradation of the structure are captured. By increasing the pressure, compaction bands throughout the sample increasingly develop and the compression curve asymptotically approaches to that of the fully degraded state of the material. At elevated pressures, the rate of compressibility will increase significantly due to particle crushing.
\end{abstract}

\section{Introduction}

Many of the natural sedimentary and residual deposits have a level of cementation that bonds the grains together and forms a structured granular medium. Apart from the source of such cementation, the bonded structure adds specific mechanical characteristics to the behaviour of the parent granular material that cannot be addressed with void ratio and stress history alone [1-4]. Intact residual soils, undisturbed soft and stiff clays, and heavily weathered rocks are examples of bounded geo-materials that their complex behaviour is affected by the presence of structure [5].

The most established method to evaluate the effect of bonds' structure on engineering behaviour is, perhaps, to examine the isotropic compression behaviour in the compression plane, i.e., the specific volume versus the mean effective stress. The effect of the structure is seen in the form of a shift in the limiting isotropic compression curve, LICC, compared to that of the parent granular geomaterial. This adds a unique permissible space to the material on the compression plane [2, 5-7]. In other words, at certain isotropic stress, the structured geomaterial pertain void ratios unachievable for the destructured state of it.

In order to evaluate the pure effect of structure on the compression behaviour and explain it within the framework of the critical state soil mechanics [8], one should compare the structured and de-structured states of the material at the same initial void ratios [9]. Such comparison can be drawn for highly porous structured geo-materials such as porous calcarenite investigated by Cuccovillo and Coop [3] or artificially bonded materials studied by Maccarini [2], Coop and Atkinson [10], Alvarado, Coop [11], and Marri, Wanatowski [12]. Moreover, Lorenzo and Bergado [13] characterized the strength and compressibility of highly porous cementtreated clays drawing the same comparison with the untreated state.

However, this method of investigation could be impossible for densely packed bonded granular geomaterials. This is mainly due to the relatively lower void ratio of the intact structured state compared with the minimum void ratio, $e_{\min }$, of the parent soil or the fully destructured state. The reason why such low initial void ratio is not achievable in the laboratory by established compaction methods can be inferred from Figure 1 which shows an SEM shot of bonds in a densely packed granular geo-material: a weak sandstone from Leura region, NSW, Australia. Such perfect particle interlocks at the microscale, cannot be regenerated by laboratory compaction at the macro scale. The Leura weak sandstone will be used later in this paper as an example of a dense but low-strength structured geo-material.

In addition to the issue of low void ratio, the other important reason that the behaviour of densely packed structured geo-materials is almost overlooked within the framework of critical state soil mechanics is the fact that such materials are usually of high strength characteristics that may render them out of the realm of soil mechanics.

\footnotetext{
${ }^{*}$ Corresponding author: $\underline{\text { m.khoshini@ } @ \text { unsw.edu.au }}$
} 
However, weak structured geo-materials are not strong enough to be studied within the established rock mechanics frameworks too.

Proper characterisation of the behaviour of densely packed but very weak structured granular geo-materials is currently lacking. This paper introduces a method to work out the conceivable de-structured state at void ratios unachievable for the parent soil, within the framework of critical state soil mechanics. This is done by quantifying the compression behaviour of the parent soil at its void ratio extremes, $e_{\max }$ and $e_{\min }$, through the definition of three parameters hereby denoted as the compression parameters. Having the relationship of compression parameters with the void ratio from $e_{\min }$ to $e_{\max }$, the parameters can be estimated beyond the range of extremes and the behaviour of the de-structured state of the material could be conceived.

\section{Isotropic compression of sands}

The isotropic compression behaviour of soils was conventionally formulated by Terzaghi, Peck [14] and then Schofield and Wroth [8]. Implementing the conventional model, the behaviour of clays under isotropic compression can be fully specified by three parameters: $\lambda, \kappa$, and $N$. On the semi-logarithmic plane of specific volume-mean effective stress (the compression plane or the $v-\ln p^{\prime}$ plane where $v=1+e$ is the specific volume with e being the void ratio), the slope of the normal consolidation line, $N C L$, and the unloadingreloading line, $U R L$, is denoted by $\lambda$ and $\kappa$, respectively. $N$ refers to the specific volume on the $N C L$ at $p^{\prime}=1$. Knowing these parameters for each clayey soil, void ratio evolution of the soil with respect to the applied pressure is predictable on the compression plane using the following equation:

$$
v=v_{0}-\kappa \ln \left(\frac{p_{c}^{\prime}}{p_{i}^{\prime}}\right)-\lambda \ln \left(\frac{p_{f}^{\prime}}{p_{c}^{\prime}}\right)
$$

where $p_{c}^{\prime}$ is the pre-consolidation pressure that can be obtained by knowing the $N C L$ and $U R L$ :

$$
p_{c}^{\prime}=e^{\left(\frac{N-v_{0}}{\lambda-\kappa}\right)}
$$

Isotropic volume change of granular geo-materials, however, does not quite follow such a simple behaviour. There is no unique $\lambda$ for a particular type of sand and the relationship between the void ratio and the mean effective stress is not linear [15-17]. The maximum and minimum void ratios, $e_{\max }$ and $e_{\min }$, for granular materials are known to be dependent on gradation, particle shape and fines content $[18,19]$ and above all, the mineralogy of the grains plays a significant role in their overall compression behaviour $[16,20]$. Therefore, even for a specific cohesionless soil, $N$ is not unique and is a function of the initial void ratio of the material or the initial relative density.

Figure 2 plots the results from numerous isotropic compression or one-dimensional compression tests regenerated from the work of many researchers $[16,20$, $21]$. The figure shows data mainly for quartz sand as the behaviour of sands of other mineralogy are significantly different and should be plotted with a separate scale. As observed, for example for the three Toyoura sand samples with the same gradation but different initial void ratios, it is rather difficult to identify a unique $N C L$. Moreover, any $N C L$ is only conceivable at high pressures at which the grading has been altered due to particle crushing.

By emerging the shortcomings of the conventional model to describe the behaviour of cohesionless soils, several researchers tried to propose mathematical models to capture the complicated compression behaviour of sands $[16,17,22,23]$, however, due to their complexities, none of the proposed models reached the popularity of the conventional model and the majority of researchers working on constitutive laws bask in the ease of implementation and adopt the conventional model for both clays and sands.

\section{Quantification of isotropic compression behaviour}

On the $v-\ln p^{\prime}$ plane, important features helpful to quantify the compression behaviour of a granular geomaterial can be listed as follows: the initial and final rate of compression from very low to escalated isotropic pressures, the transition between these two extreme states, and the isotropic pressure at which the particle breakage becomes prominent, $p_{c r}^{\prime}$.

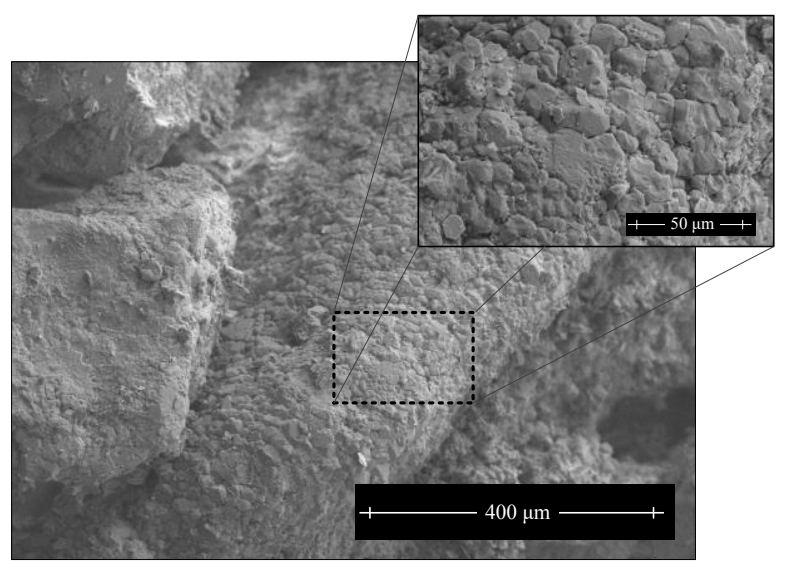

Fig. 1. SEM photo of bonds in Leura weak sandstone

The ratio of $R_{\lambda}=\lambda_{0} / \lambda_{c r}$ can be defined where $\lambda_{0}$ and $\lambda_{c r}$ are the initial and final slope of the compression curve, respectively, obtained at very low isotropic pressures (up to $1 \mathrm{MPa}$ ) and at escalated pressures at which particle crushing is dominant. The point of the maximum curvature of the compression curve also indicates the $p_{c r}^{\prime}$. The value of the maximum curvature describes the abruptness of the change in the compression rate around $p_{c r}^{\prime}$. 


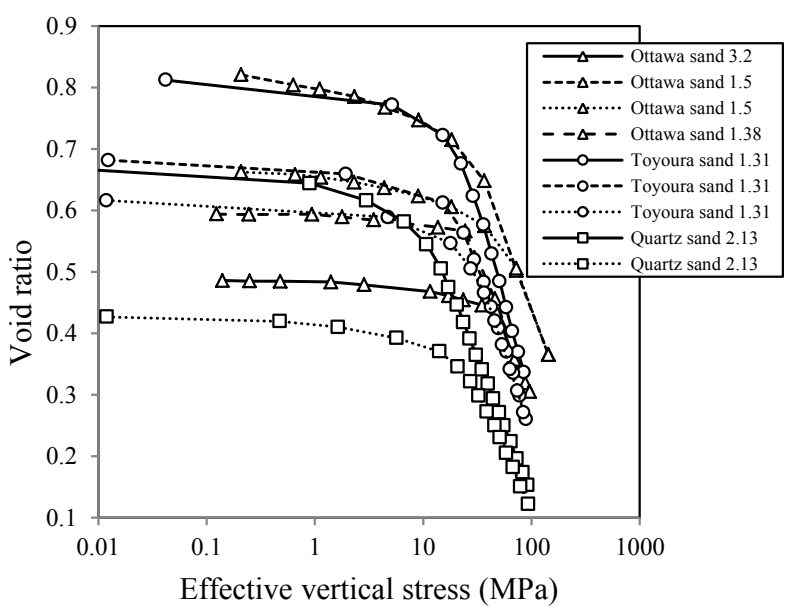

Fig. 2. Compression curves for several quartz sands

\section{Effective parameters on the compressibility of sands}

Mineralogy of the grains, gradation of the granular material, and the relative density at which the material initially undergoes the pressure are known to be the main effective parameters on the compressibility of granular geo-materials $[16,21,24]$. These parameters are discussed in the following sections.

Mineralogy defines the strength of every single particle against crushing. The domain of compressibility and the scale of pressures at which the rate of compressibility abruptly changes are different for granular materials of different mineralogy. The initial and final rate of compression and the transition between them from very low to escalated pressures are reported to be different for sands of almost similar gradation. Sands of different composition i.e. quartz, feldspar, carbonate, mica, and chert exhibit very different behaviours [20]. It is reported that $\mathrm{p}_{\mathrm{cr}}^{\prime}$ is generally higher for quartz sands, and carbonate and feldspar sands demonstrate the lowest $p_{c r}^{\prime}$ [25]. Such particle crushing behaviour explains the higher compressibility of feldspar and carbonate sands rendering larger absolute values for $\lambda_{0}$. However, all sand types tend to have almost similar $\lambda_{\text {cr }}$ at high enough effective vertical stresses.

Gradation is a prominent factor controlling the compression behaviour of sands. Data from [21] on angular silica sand mixtures covering a wide range of gradation $(\mathrm{Cu}$ from 1.1 to 9.9) shows the significant effect of gradation on $\mathrm{p}_{\mathrm{cr}}^{\prime}, \lambda_{0}, \lambda_{\mathrm{cr}}$, and eventually $\mathrm{R}_{\lambda}$.

Fines content (both plastic and non-plastic) is also known to affect the compression behaviour of sands. Fine contents are considered isotropically non-crushable and change the gradation of the parent sand towards higher $C_{u}$ 's. Furthermore, the fines content changes the soil behaviour through changing the characterisation of chain forces.

Relative density or the initial void ratio at which the isotropic compression starts affects $p_{c r}^{\prime}$ and $\lambda_{0}$ substantially. Data from [26] on a well-graded quartz sand $\left(C_{u}=2.4\right.$ and $\left.D_{50}=0.20 \mathrm{~mm}\right)$ shows that $p_{c r}^{\prime}$ and $\lambda_{0}$ vary from 0.0038 to 0.0067 and 16.11 to $13.03 \mathrm{MPa}$, respectively, for $D_{r}=37 \%$ and $77 \%$.

\section{Isotropic compression of a dense structured granular geo-material}

The behaviour of the structured granular geo-materials is discussed in this section. The framework for quantification of the behaviour of unstructured granular geomaterials under isotropic pressure discussed in the previous sections is used to propose a procedure for estimation of the behaviour of the conceived de-structured material at the initial void ratios pertinent to the structured state of the material for cases where the initials void ratio is lower than $e_{\min }$ of the parent materials. It is suggested that the variation of the compression parameters of the parent sand are obtained with respect to the initial void ratio of the parent sand over the range of $e_{\min }$ to $e_{\max }$. This requires performing isotropic compression tests on samples of the parent soil at various initial densities. Once such a variation is obtained, it can be extrapolated to estimate the compression parameters at initial void ratios lower than $e_{m i n}$. This procedure will be explained in details in the following sections. The Leura weak sandstone is taken as an example and the proposed procedure is implemented for it.

\section{Material properties and testing procedure}

The Leura weak sandstone is used as an example of dense but low-strength structured geo-material in this study. The rock formation is generally known as the Banks Wall sandstone which is white to amber coloured with visible bedding layers and a grainy rough surface that is highly weathered at shallow depths, very friable, and susceptible to mechanical and moisture degradation. The Leura weak rock has a natural void ratio ranging from 0.25 to 0.36 with a mean value of 0.32 and an average dry unit weight of $19.92 \mathrm{kN} / \mathrm{m}^{3}$. The average unconfined compression strength is obtained as $2.75 \mathrm{MPa}$ that indicates the material is categorized as R1-grade, very weak rock based on [27].

The fully de-structured state of the weak rock is obtained by gently crushing lumps of the intact rock into its constituent particles with a rubber pestle and mortar. The gradation of the de-structured (remoulded) state of the weak rock can be described as well graded sand with $10-15 \%$ fines (mainly kaolinite). The coefficient of uniformity, $C_{u}$, is 7.1 and the coefficient of curvature, $C_{c}$, is 1.53 . The maximum and minimum void ratio of the remoulded material is measured as 0.88 and 0.39 , respectively.

Intact cylindrical samples of the weak sandstone were obtained for the isotropic compression tests in low and high-pressure triaxial cells. The low-pressure triaxial cell used in this research is mainly designed for soil testing with a maximum capacity of $3.5 \mathrm{MPa}$ isotropic pressure. The cell is equipped with a hydraulic pressure controller which is accurate to $\pm 1 \%$ of the full capacity. The back pressure controller is capable of measuring the volume change during each stage of the test with a precision of \pm 1 $\mathrm{mm}^{3}$. Axial displacement of the sample was measured using a linear potentiometer displacement transducer 
(LPDT) with a maximum range of $50 \mathrm{~mm}$ and precision of $\pm 10^{-4} \mathrm{~mm}$. The high-pressure triaxial cell used in this research is a new generation of Hoek cell equipped with four local LVDTs to measure the radial deformations of the test sample. The cell operates under the confining pressures up to $130 \mathrm{MPa}$. The axial deformation was measured using the frame axial displacement. An unconventional $0.9 \mathrm{~mm}$ thick rubber sleeve made from flexible M-grade Viton material was used in order to precisely measure the radial deformation [28].

Test samples of intact and de-structured weak rock were tested under isotropic stress path from nil to up to $2.5 \mathrm{MPa}$ for low-pressure tests and up to $100 \mathrm{MPa}$ for high-pressure tests. The volumetric strain was calculated based on the measured axial and radial strains and used to work out the void ratio of the sample at each corresponding pressure. The results were then plotted on the compression plane for both intact and de-structured state of the material.

\section{Test results and discussion}

\subsection{Parent soil}

The specific volume versus the mean effective stress is plotted on a semi-logarithmic plane for re-moulded samples of the parent soil at $D_{r}=94 \%, 53 \%$, and $10 \%$ in Figure 3. Separate test data for low and high-pressure tests are plotted together: the solid lines indicate low-pressure tests at which the sample is unloaded as well and the dotted lines with data points indicate high-pressure test results. Compression parameters of $\mathrm{p}_{\mathrm{cr}}^{\prime}, \lambda_{0}, \lambda_{\mathrm{cr}}$, and $\mathrm{R}_{\lambda}$ are obtained as $8.4 \mathrm{MPa}, 0.017,0.196$, and 0.076 for the loosest and 10.4 MPa, 0.008, 0.084, and 0.095 for the densest sample, respectively. These parameters, as expected, are very close to those of well-graded sands with high $C_{u}$ 's or mixed sands with $10-15 \%$ fines content reported in the literature [20,21]. The slope of un-loading reloading line, $\kappa$, is measured as 0.0044 and 0.0087 for dense and loose samples, respectively.

\subsection{Structured soil}

For the structured state, plotting the specific volume against the mean effective stress shows an initial slope, $\lambda_{0 s t r}$, considerably smaller than the corresponding slope for the de-structured material. The material continues to demonstrate such a low compressibility as the mean effective stress increases up to a point where bond breakage is initiated and the compression rate starts to increase. This is called the onset of structure collapse and the corresponding pressure is denoted as $p_{c s-i}^{\prime}$. The results of the test on the structured soil are plotted in Figure 3 to draw a comparison with the parent sand samples and also plotted separately in Figure 5 on a better scale to illustrate the compression parameters.

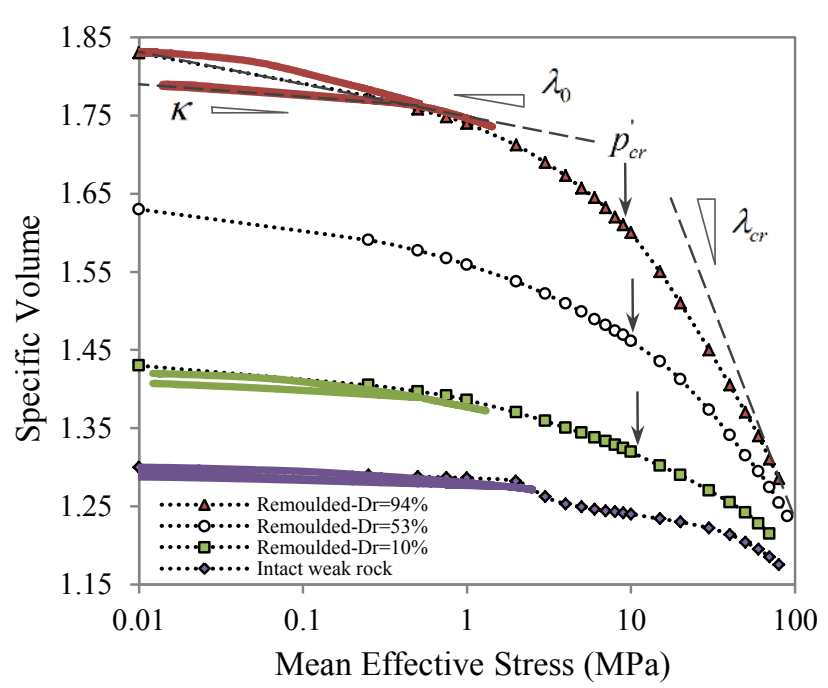

Fig. 3. Compression behaviour of the Leura weak sandstone as a densely packed but weak structured geo-material.

It should be noted that the structure collapse under isotropic loading is not an instantaneous phenomenon. There is an onset and a completion for it whose corresponding pressures for the latter is hereby denoted as $p_{c s-f}^{\prime}$. The onset of the structure collapse is considered when the first group of bonds crack and exit the stress chain throughout the material as the isotropic pressure increases. It could instigate a progressive collapse under the constant pressure but does not necessarily result in a complete elimination of the bonds. As the pressure increases more, stress is transferred throughout the force chain and more bonds fail. At the completion of the structure collapse, theoretically, stress is transferred between the granular particles by pure friction. Compression parameters for the structured state are defined as $p_{c s-i}^{\prime}, \lambda_{0 s t r}, \lambda_{c r}$, and $\kappa$, as shown in Figure 5 -a. These parameters are measured as $1.98 \mathrm{MPa}, 0.003$, 0.075 , and 0.0021 respectively for the sample tested.

The position of $p_{c s-f}^{\prime}$, for now, is rather vague as it happens at elevated isotropic pressures where all bonds are degraded. At such high pressures, the particle breakage is a dominant phenomenon, therefore, any change in the curvature of the compression curve cannot be solely associated with the bond breakage but is a function of particle breakage too. Having an estimation of the behaviour of the parent sand isotropically compressed from the same initial void ratio as the structured state does, makes it possible to identify $\mathrm{p}_{\mathrm{cs}-\mathrm{f}}^{\prime}$.

As can be seen in Figure 3, the initial void ratio of the intact material is lower than $e_{\text {min }}$ of the parent sand $(0.30$ versus 0.39 respectively). Therefore, the behaviour of the fully de-structured state at the initial void ration of the structured material has to be estimated. 
Figure 4-a plots the variation of $\lambda_{0}$ with the initial specific volume of the parent sand, obtained from performing three isotropic compression tests on three samples of the parent sand with different initial void ratios. Apart from the three data points obtained from experiments, one more data point can be assumed given that at the limit of $v=1.0, \lambda_{0}$ must be zero. Assuming a logarithmic relationship as the best fit function, $\lambda_{0}$ of the de-structured state at $v=1.30$ can be estimated as 0.007 . Considering that the data on parent sand samples do not reach the $\lambda_{c r}$, in order to estimate the slope of isotropic compression behaviour at elevated pressures, a mean effective stress of $80 \mathrm{MPa}$, at which the data for parent sand is available, is selected and the relationship of $\lambda_{80 \mathrm{MPa}}$ with specific volume is then developed in a fashion similar to what explained for $\lambda_{0}$. As a result, $\lambda_{80 \mathrm{MPa}}$ of the de-structured state at $v=1.30$ is estimated as 0.077 (Figure 4-b). Finally, the specific volume at mean effective stress of $80 \mathrm{MPa}$ is estimated as $v=1.17$ based on the data available for parent sand plotted in Figure 4c. Having both the value and first derivative of specific volume with respect to the mean effective stress at two points $\left(p^{\prime}=10 \mathrm{kPa}\right.$ and $\left.80 \mathrm{MPa}\right)$, the compression behaviour for the de-structured state of the material is estimated and plotted in Figure 5-b for the initial void ratio of interest. As can be seen, structured and destructured state behaviours tend to merge as the pressure surpasses $80 \mathrm{MPa}$. This helps correct estimation of $p_{c s-f}^{\prime}$ as well as the structure-permitted area on the compression plane.

\section{Conclusion}

The isotropic compression behaviour of cohesionless granular geo-materials was explained and important parameters affecting their behaviour were introduced as mineralogy, gradation, and relative density. In order to quantify the behaviour, compression parameters were defined and then the effect of mineralogy, gradation, and relative density on them was discussed.

The role of structure in the isotropic compression behaviour of the material was then explained, which involves an initial lower compressibility followed by a collapse that increases the rate of volume shrinkage. This adds a permissible area on the compression plane allowing the material to retain higher void ratios under the same isotropic pressures compared to the de-structured stage.

A procedure was then proposed to estimate the compression behaviour of the de-structured state of the material at the same initial void ratio as the structured state since this is required to correctly capture the effect of structure. The procedure proposed was successfully implemented to characterise compression behaviour of a weak sandstone, the Leura weak sandstone which is a very dense but low-strength structured geo-material.
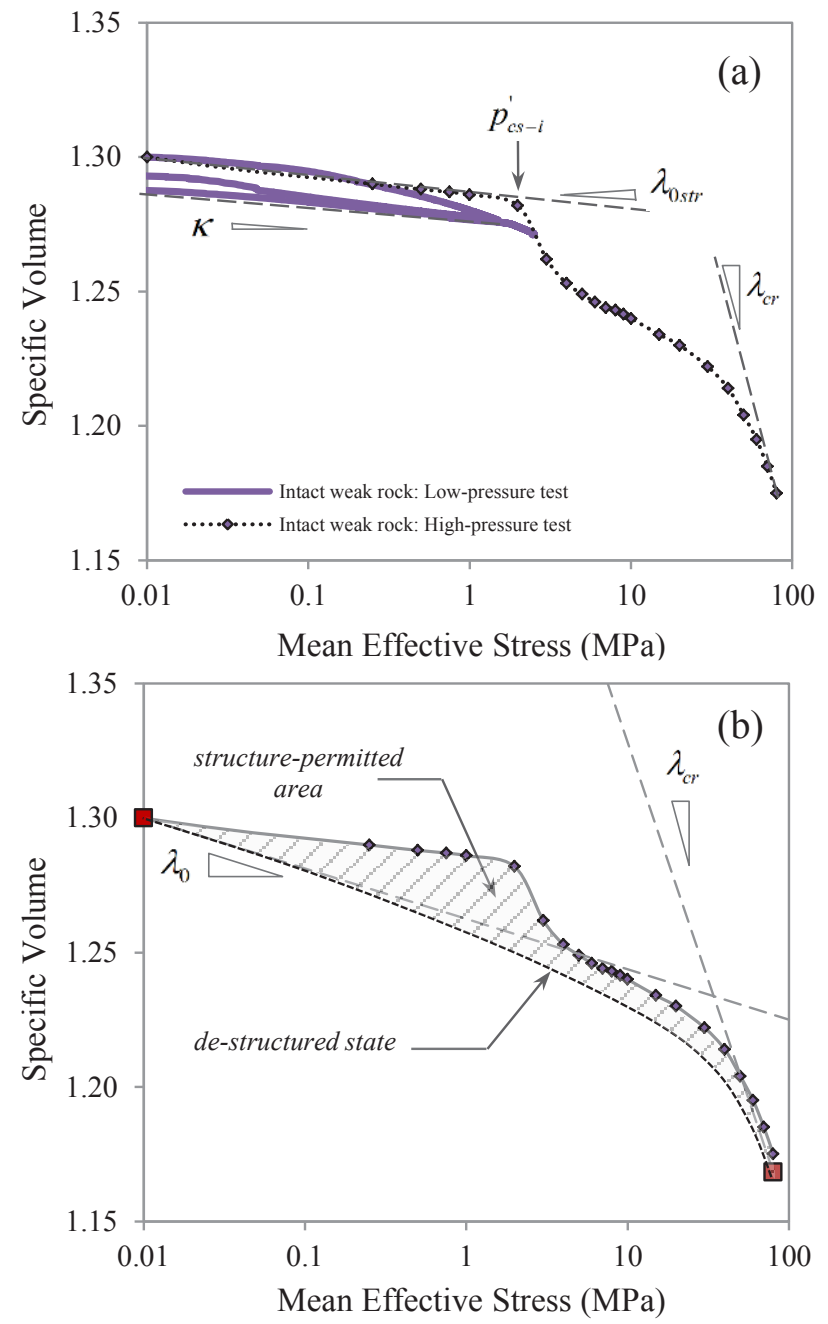

Fig. 5. Leura weak sandstone as a structured geo-material: (a) isotropic compression behaviour of the intact structured state and (b) estimating the isotropic compression behaviour of the de-structured state
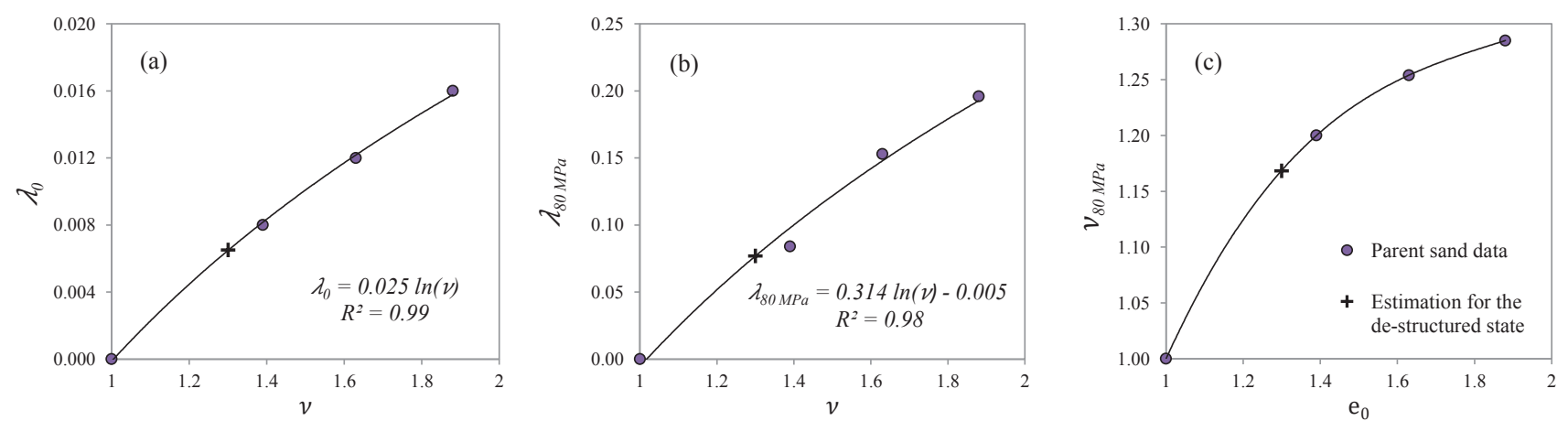

Fig. 4. Deriving parameters needed to estimate the behaviour of the de-structured state of the material 


\section{References}

1. Sangrey, D., Naturally cemented sensitive soils. Geotechnique, 1972. 22(1): p. 139-152.

2. Maccarini, M., Laboratory studies for a weakly bonded artificial soil. 1987.

3. Cuccovillo, T. and M.R. Coop, Yielding and prefailure deformation of structured sands. Géotechnique, 1997. 47(3): p. 491-508.

4. Amorosi, A. and S. Rampello, An experimental investigation into the mechanical behaviour of a structured stiff clay. Géotechnique, 2007. 57(2): p. 153-166.

5. Leroueil, S. and P.R. Vaughan, The general and congruent effects of structure in natural soils and weak rocks. Géotechnique, 1990. 40(3): p. 467-488.

6. Lagioia, R. and R. Nova, An experimental and theoretical study of the behaviour of a calcarenite in triaxial compression. Géotechnique, 1995. 45(4): p. 633-648.

7. Cuccovillo, T. and M.R. Coop, On the mechanics of structured sands. Géotechnique, 1999. 49(6): p. 741-760.

8. Schofield, A. and P. Wroth, Critical state soil mechanics. Vol. 310. 1968: McGraw-Hill London.

9. Wesley, L.D., Discussion: Structural behaviour of residual soils of the continually wet Highlands of Papua New Guinea. Géotechnique, 1974. 24(1): p. 101-106.

10. Coop, M. and J. Atkinson, The mechanics of cemented carbonate sands. Geotechnique, 1993. 43(1): p. 53-67.

11. Alvarado, G., M.R. Coop, and S. Willson, On the role of bond breakage due to unloading in the behaviour of weak sandstones. Géotechnique, 2012. 62(4): p. 303-316.

12. Marri, A., D. Wanatowski, and H. Yu, Drained behaviour of cemented sand in high pressure triaxial compression tests. Geomechanics and Geoengineering, 2012. 7(3): p. 159-174.

13. Lorenzo, G.A. and D.T. Bergado, Fundamental parameters of cement-admixed clay-New approach. Journal of geotechnical and geoenvironmental engineering, 2004. 130(10): p. $1042-1050$.

14. Terzaghi, K., R.B. Peck, and G. Mesri, Soil mechanics in engineering practice. 1996: John Wiley \& Sons.

15. Been, K., M. Jefferies, and J. Hachey, Critical state of sands. Geotechnique, 1991. 41(3): p. 365-381.

16. Pestana, J.M. and A. Whittle, Compression model for cohesionless soils. Géotechnique, 1995. 45(4): p. 611-631.

17. Jefferies, M. and K. Been, Implications for critical state theory from isotropic compression of sand. Géotechnique, 2000. 50(4): p. 419-429.

18. Lade, P.V., C. Liggio, and J.A. Yamamuro, Effects of non-plastic fines on minimum and maximum void ratios of sand. Geotechnical testing journal, 1998. 21: p. 336-347.

19. Cubrinovski, M. and K. Ishihara, Maximum and minimum void ratio characteristics of sands. Soils and foundations, 2002. 42(6): p. 65-78.

20. Chuhan, F.A., et al., Experimental compression of loose sands: relevance to porosity reduction during burial in sedimentary basins. Canadian Geotechnical Journal, 2003. 40(5): p. 995-1011.

21. Nakata, Y., et al., Microscopic particle crushing of sand subjected to high pressure onedimensional compression. Soils and foundations, 2001. 41(1): p. 69-82.

22. Hardin, B.O., 1-D strain in normally consolidated cohesionless soils. Journal of Geotechnical Engineering, 1987. 113(12): p. 1449-1467.

23. Meidani, M., C.S. Chang, and Y. Deng, On active and inactive voids and a compression model for granular soils. Engineering Geology, 2017. 222: p. 156-167.

24. Mesri, G. and B. Vardhanabhuti, Compression of granular materials. Canadian Geotechnical Journal, 2009. 46(4): p. 369-392.

25. Nakata, A., et al., A probabilistic approach to sand particle crushing in the triaxial test. Géotechnique, 1999. 49(5): p. 567-583.

26. Yet, N.S., Time-dependent behavior of piles in sand. 1998, National University of Singapore: Singapore.

27. Marinos, P. and E. Hoek, Estimating the geotechnical properties of heterogeneous rock masses such as flysch. Bulletin of engineering geology and the environment, 2001. 60(2): p. 8592.

28. Regenauer-Lieb, K., et al., New Generation of Hoek Cells. Geotechnical Testing Journal, 2018. 42(3). 